\title{
EXISTENCE OF RESOLVENT FOR VOLTERRA INTEGRAL EQUATIONS ON TIME SCALES
}

\author{
MURAT ADIVAR ${ }^{\bowtie}$ and YOUSSEF N. RAFFOUL
}

(Received 5 November 2009)

\section{Abstract}

We introduce the concept of 'shift operators' in order to establish sufficient conditions for the existence of the resolvent for the Volterra integral equation

$$
x(t)=f(t)+\int_{t_{0}}^{t} a(t, s) x(s) \Delta s, \quad t_{0} \in \mathbb{T}^{\kappa},
$$

on time scales. The paper will serve as the foundation for future research on the qualitative analysis of solutions of Volterra integral equations on time scales, using the notion of the resolvent.

2000 Mathematics subject classification: primary 45D05; secondary 39A12.

Keywords and phrases: existence, resolvent, shift operator, time scales, Volterra integral equation.

\section{Introduction}

In [10], the existence of a resolvent kernel $r(t, s)$ corresponding to integral equations of the form

$$
x(t)=f(t)+\int_{0}^{t} a(t, s) x(s) d s
$$

is discussed by giving several theorems that provide sufficient conditions on $a(t, s)$. In this paper, we extend the theory established in [10] to the Volterra integral equation

$$
x(t)=f(t)+\int_{t_{0}}^{t} a(t, s) x(s) \Delta s,
$$

on time scales, in which integral, summation, and $q$-integral equations are included as special cases. There are few papers that deal with the qualitative theory of solutions of integral equations on time scales. So far as the present authors are aware, the paper of Kulik and Tisdell [9] is the only one on integral equations on time scales that deals with

This work was supported by the Scientific and Technological Research Council of Turkey.

(c) 2010 Australian Mathematical Publishing Association Inc. 0004-9727/2010 \$16.00 
guaranteeing the existence, uniqueness, and boundedness of solutions. In particular, Kulik and Tisdell [9, p. 105] claim that "the importance of integral equations on time scales is clear: there are no dynamic equations on time scales, there are only integral equations on time scales' as 'in the investigation of dynamic equations on time scales, the analysis most often turns to that of a related integral equation on time scales'.

In the continuous case, there are volumes written on integral equations where either the Lyapunov functional or the notion of the resolvent is used to obtain results regarding the existence of solutions, periodic solutions and stability. The resolvent for integral equations on time scales has not been developed and the authors intended to do so in this paper. However, during the preparation of this paper the authors realized that, in order to prove the continuity of the resolvent, they had to require $t-s$ or $t+s$ to be in the time scale. This unpleasant restriction led to the idea of 'shift operators', which we state in Definition 3.9. The authors will make use of the shift operators in future work where Lyapunov functionals are used to prove existence of solutions of integral equations on time scales. Thus, the notion of shift operators goes beyond this paper and will have wider positive implications for the general theory of time scales.

A time scale is a nonempty closed subset of real numbers. The delta derivative of a function $f$, defined by

$$
f^{\Delta}(t):=\lim _{s \rightarrow t} \frac{f(\sigma(t))-f(s)}{\sigma(t)-s} \quad \text { where } s \rightarrow t, s \in \mathbb{T} \backslash\{\sigma(t)\},
$$

was first introduced by Hilger [7] to unify discrete and continuous analyses. In (1.1), $\sigma: \mathbb{T} \rightarrow \mathbb{T}$ is the forward jump operator defined by $\sigma(t):=\inf \{s \in \mathbb{T}: s>t\}$. Evidently,

$$
\sigma(t)= \begin{cases}t & \text { if } \mathbb{T}=\mathbb{R} \\ t+1 & \text { if } \mathbb{T}=\mathbb{Z}\end{cases}
$$

and hence the delta derivative turns into an ordinary derivative if $\mathbb{T}=\mathbb{R}$ and it becomes the forward difference operator for $\mathbb{T}=\mathbb{Z}$. It is evident from various papers (see $[1,5,6,12]$ and references therein) that dynamic equations on time scales are useful tools to explain the differences and similarities between difference and differential equations. Providing a wide perspective, the theory of time scales also leads to some new results for both the discrete and continuous cases. Furthermore, since there are many time scales which are different from $\mathbb{R}$ and $\mathbb{Z}$, investigation of dynamic equations on time scales constitutes a more general and comprehensive theory.

We assume that the reader is familiar with the basic calculus of time scales. A comprehensive review on $\Delta$-derivative and $\Delta$-Riemann integrals can be found in [5].

Henceforth we denote by $[a, b]_{\mathbb{T}}$ the closed interval $[a, b] \cap \mathbb{T}$ of the time scale $\mathbb{T}$. The intervals $[a, b)_{\mathbb{T}},(a, b]_{\mathbb{T}}$, and $(a, b)_{\mathbb{T}}$ are defined similarly. A point $t \in \mathbb{T}$ is said to be right dense (left dense) if $\sigma(t)=t(\sup \{s \in \mathbb{T}: s<t\}=t)$. A function $f: \mathbb{T} \rightarrow \mathbb{R}$ is called $r d$-continuous if it is continuous at right dense points and its leftsided limits exist (finite) at left dense points. Every $r d$-continuous function $f: \mathbb{T} \rightarrow \mathbb{R}$ 
has an anti-derivative

$$
F(t)=\int_{t_{0}}^{t} f(t) \Delta t .
$$

That is, $F^{\Delta}(t)=f(t)$ for all $t \in \mathbb{T}^{\kappa}:=\mathbb{T} \backslash\{\max \mathbb{T}\}$ (see [6, Theorem 1.27]).

In [2, pp. 157-163] a theory for a Lebesgue $\Delta$-integration is established by means of Lebesgue $\Delta$-measure, denoted $\mu_{\Delta}$, with the following properties.

THEOREM 1.1 [2, Theorem 5.76]. For each $s_{0} \in \mathbb{T}^{\kappa}$, the single point set $\left\{s_{0}\right\}$ is $\Delta$ measurable, and its measure is given by

$$
\mu_{\Delta}\left\{s_{0}\right\}=\sigma\left(s_{0}\right)-s_{0}=\mu\left(s_{0}\right),
$$

where $\mu: \mathbb{T} \rightarrow[0, \infty)$ is the step size function defined by $\mu(t):=\sigma(t)-t$.

TheOREM 1.2 [2, Theorem 5.77]. If $a, b \in \mathbb{T}$ and $a \leq b$, then

$$
\mu_{\Delta}\left\{[a, b)_{\mathbb{T}}\right\}=b-a, \quad \mu_{\Delta}\left\{(a, b)_{\mathbb{T}}\right\}=b-\sigma(a) .
$$

If $a, b \in \mathbb{T}^{\kappa}$ and $a \leq b$, then

$$
\mu_{\Delta}\left\{(a, b]_{\mathbb{T}}\right\}=\sigma(b)-\sigma(a), \quad \mu_{\Delta}\left\{[a, b]_{\mathbb{T}}\right\}=\sigma(b)-a .
$$

Furthermore, it is concluded in [2, p. 159] that all theorems of the general Lebesgue integral theory, including the Lebesgue dominated convergence theorem, also hold for the Lebesgue $\Delta$-integral on time scales. The next theorem gives the relationship between Riemann and Lebesgue integrals on time scales.

THEOREM 1.3 [2, Theorem 5.81]. Let $f$ be a bounded real-valued function defined on $[a, b]_{\mathbb{T}}$. If $f$ is Riemann $\Delta$-integrable from a to $b$, then $f$ is Lebesgue $\Delta$-integrable on $[a, b)$ and

$$
\int_{a}^{b} f(t) \Delta t=\int_{[a, b)} f \mu_{\Delta} .
$$

DEFINITION 1.4. We say that a property holds almost everywhere (a.e.) in $S \subset \mathbb{T}$ if the set of all right dense points of $S$ for which the property does not hold is a null set, that is, is a set with $\Delta$-measure zero.

The following theorem gives a criterion for $\Delta$-Riemann integrability of a function.

THEOREM 1.5. Let $f$ be a bounded function defined on the finite closed interval $[a, b]_{\mathbb{T}}$ of the time scale $\mathbb{T}$. Then $f$ is Riemann $\Delta$-integrable from $a$ to $b$ if and only if $f$ is continuous a.e. in $[a, b)_{\mathbb{T}}$.

Let $\mathbb{T}_{1}$ and $\mathbb{T}_{2}$ be two time scales. In [4], the double $\Delta$-integral of a multivariable function $f: \mathbb{T}_{1} \times \mathbb{T}_{2} \rightarrow \mathbb{R}$ over a rectangular region $[a, b)_{\mathbb{T}_{1}} \times[c, d)_{\mathbb{T}_{2}}$ is introduced. The following result provides sufficient conditions for interchanging the order of integration over a rectangular region. 
LEMMA 1.6 [4, Remark 2.17]. Let $f$ be $\Delta$-integrable over $R=[a, b)_{\mathbb{T}_{1}} \times[c, d)_{\mathbb{T}_{2}}$ and suppose that the single integrals

$$
\begin{aligned}
& K(y)=\int_{a}^{b} f(x, y) \Delta_{1} x, \\
& I(x)=\int_{c}^{d} f(x, y) \Delta_{2} y
\end{aligned}
$$

exists for each $x \in[a, b)_{\mathbb{T}_{1}}$ and $y \in[c, d)_{\mathbb{T}_{2}}$. Then

$$
\begin{aligned}
\iint_{R} f(x, y) \Delta_{1} x \Delta_{2} y & =\int_{a}^{b} \Delta_{1} x \int_{c}^{d} f(x, y) \Delta_{2} y \\
& =\int_{c}^{d} \Delta_{2} y \int_{a}^{b} f(x, y) \Delta_{1} x
\end{aligned}
$$

where $\Delta_{1}$ and $\Delta_{2}$ denote the derivatives on $\mathbb{T}_{1}$ and $\mathbb{T}_{2}$, respectively.

Multiple $\Delta$-integration over more general sets is defined in [3, Definitions 4.13, 4.15].

TheOrem 1.7 [3, Theorem 4.30]. Let $\varphi:[a, b]_{\mathbb{T}_{1}^{\kappa}} \rightarrow \mathbb{T}_{2}^{\kappa}$ and $\psi:[a, b]_{\mathbb{T}_{1}^{\kappa}} \rightarrow \mathbb{T}_{2}^{\kappa}$ be two continuous functions such that $\varphi(t)<\psi(t)$ for all $t \in[a, b]_{\mathbb{T}_{1}^{\kappa}}$. Let $E$ be $a$ bounded set in $\mathbb{T}_{1} \times \mathbb{T}_{2}$ given by

$$
E=\left\{(t, s) \in \mathbb{T}_{1} \times \mathbb{T}_{2}: a \leq t<b, \varphi(t) \leq s<\psi(t)\right\} .
$$

Then $E$ is Jordan $\Delta$-measurable, and if $f: E \rightarrow \mathbb{R}$ is $\Delta$-integrable over $E$ if the single integral

$$
\int_{\varphi(t)}^{\psi(t)} f(t, s) \Delta_{2} s
$$

exists for each $t \in[a, b)_{\mathbb{T}_{1}}$, then the iterated integral

$$
\int_{a}^{b} \Delta_{1} t \int_{\varphi(t)}^{\psi(t)} f(t, s) \Delta_{2} s
$$

exists and

$$
\iint_{E} f(t, s) \Delta_{1} t \Delta_{2} s=\int_{a}^{b} \Delta_{1} t \int_{\varphi(t)}^{\psi(t)} f(t, s) \Delta_{2} s .
$$

Linearity and additivity properties of multiple integrals on time scales can be found in $[3,4]$.

To differentiate the iterated integrals given in later sections we will employ the following theorem. 
THEOREM 1.8 [5, Theorem 1.117]. Let $a \in \mathbb{T}^{\kappa}, b \in \mathbb{T}$ and assume that $k: \mathbb{T} \times \mathbb{T}^{\kappa} \rightarrow$ $\mathbb{R}$ is continuous at $(t, t)$, where $t \in \mathbb{T}^{\kappa}$ with $t>a$. Also assume that $k^{\Delta}(t,$.$) is$ $r d$-continuous on $[a, \sigma(t)]$. Suppose that for each $\varepsilon>0$ there exists a neighborhood $U$ of $t$, independent of $\tau \in\left[t_{0}, \sigma(t)\right]$, such that

$$
\left|k(\sigma(t), \tau)-k(s, r)-k^{\Delta}(t, \tau)(\sigma(t)-s)\right| \leq \varepsilon|\sigma(t)-s|
$$

for all $s \in U$, where $k^{\Delta}$ denotes the derivative of $k$ with respect to the first variable. Then

$$
\begin{aligned}
g(t) & :=\int_{a}^{t} k(t, \tau) \Delta \tau \text { implies } g^{\Delta}(t)=\int_{a}^{t} k^{\Delta}(t, \tau) \Delta \tau+k(\sigma(t), t), \\
h(t) & :=\int_{t}^{b} k(t, \tau) \Delta \tau \text { implies } g^{\Delta}(t)=\int_{t}^{b} k^{\Delta}(t, \tau) \Delta \tau-k(\sigma(t), t) .
\end{aligned}
$$

\section{Construction of the resolvent equation}

In this section we use some properties of multiple $\Delta$-integrals to construct the resolvent equations corresponding to linear and nonlinear systems of integral equations on time scales.

Let $t_{0} \in \mathbb{T}^{\kappa}$ be a fixed point and let $T>t_{0}$ be given. Denote by $I_{\mathbb{T}}$ the closed interval $\left[t_{0}, T\right]_{\mathbb{T}}$. For our future computations, it is essential to know when the formula

$$
\begin{aligned}
\iint_{E_{1}} f(s, u) \Delta s \Delta u & =\int_{t_{0}}^{t} \Delta u \int_{t_{0}}^{u} f(s, u) \Delta s \\
& =\int_{t_{0}}^{t} \Delta s \int_{\sigma(s)}^{t} f(s, u) \Delta u, \quad t \in I_{\mathbb{T}},
\end{aligned}
$$

holds. Here, $E_{1}$ is the triangular region given by

$$
E_{1}:=\left\{(s, u) \in I_{\mathbb{T}} \times I_{\mathbb{T}}: t_{0} \leq s<u, t_{0} \leq u<t\right\} .
$$

Evidently, $E_{1}$ is a bounded subset of $\left[t_{0}, T\right)_{\mathbb{T}} \times\left[t_{0}, T\right)_{\mathbb{T}}$.

Let $t \in I_{\mathbb{T}}$ be fixed. For the existence of iterated integrals in (2.1) it is natural to require that all integrals

$$
K(u):=\int_{t_{0}}^{u} f(s, u) \Delta s, \quad J(s, t):=\int_{\sigma(s)}^{t} f(s, u) \Delta u
$$

exist for each $u \in\left[t_{0}, t\right)_{\mathbb{T}}$ and $s \in\left[t_{0}, t\right)_{\mathbb{T}}$.

Inspired by the method in [8, Lemma 1], we obtain the following result which enables us to interchange the order of integration over a triangular region.

LEMma 2.1. Suppose that $f: I_{\mathbb{T}} \times I_{\mathbb{T}} \rightarrow \mathbb{R}$ is $\Delta$-integrable over $E_{1}$ and that the single integrals (2.3) exist for each $u \in\left[t_{0}, t\right)_{\mathbb{T}}$ and $s \in\left[t_{0}, t\right)_{\mathbb{T}}$. If the function 
$J(s, t)$ defined in (2.3) satisfies the conditions of Theorem 1.8, then (2.1) holds for all $t \in I_{\mathbb{T}}$.

Proof. First, from (2.2) and Theorem 1.7 we deduce that the set $E_{1}$ is Jordan $\Delta$ measurable and the double integral

$$
\iint_{E_{1}} f(s, u) \Delta s \Delta u
$$

exists. The existence of single integrals in (2.3) and Theorem 1.7 imply existence of iterated integrals and the equality

$$
\iint_{E_{1}} f(s, u) \Delta s \Delta u=\int_{t_{0}}^{t} \Delta u \int_{t_{0}}^{u} f(s, u) \Delta s, \quad t \in I_{\mathbb{T}} .
$$

Theorem 1.8 guarantees that the function

$$
h(t):=\int_{t_{0}}^{t} \Delta u \int_{t_{0}}^{u} f(s, u) \Delta s-\int_{t_{0}}^{t} \Delta s \int_{\sigma(s)}^{t} f(s, u) \Delta u, \quad t \in I_{\mathbb{T}},
$$

is $\Delta$-differentiable and

$$
\begin{aligned}
h^{\Delta}(t) & =\int_{t_{0}}^{t} f(s, t) \Delta s-\int_{\sigma(t)}^{\sigma(t)} f(t, u) \Delta u-\int_{t_{0}}^{t} \Delta s\left[\int_{\sigma(s)}^{t} f(s, u) \Delta u\right]^{\Delta_{t}} \\
& =\int_{t_{0}}^{t} f(s, t) \Delta s-\int_{t_{0}}^{t} f(s, t) \Delta s=0 \quad \forall t \in\left[t_{0}, T\right)_{\mathbb{T}} .
\end{aligned}
$$

Applying [6, Corollary 1.15] we conclude that $h$ is constant. On the other hand, since $h\left(t_{0}\right)=0$, then $h(t)=0$ for all $t \in I_{\mathbb{T}}$. The proof is complete.

First, we consider the linear system of integral equations of the form

$$
x(t)=f(t)+\int_{t_{0}}^{t} a(t, s) x(s) \Delta s .
$$

The corresponding resolvent equation associated with $a(t, s)$ is given by

$$
r(t, s)=-a(t, s)+\int_{\sigma(s)}^{t} r(t, u) a(u, s) \Delta u .
$$

If $a$ is scalar-valued, then so is $r$. If $a$ is an $n \times n$ matrix, then so is $r$.

If Equation (2.5) has a solution $r(t, s)$ and we can justify the use of Lemma 2.1 along with all necessary integrals, then the solution of the linear system (2.4) may be written in terms of $f$ as follows:

$$
x(t)=f(t)-\int_{t_{0}}^{t} r(t, u) f(u) \Delta u
$$


To see this we multiply both sides of (2.4) by $r(t, s)$ to obtain

$$
\begin{aligned}
\int_{t_{0}}^{t} r(t, u) x(u) \Delta u-\int_{t_{0}}^{t} r(t, u) f(u) \Delta u & =\int_{t_{0}}^{t} r(t, u) \int_{t_{0}}^{u} a(u, s) x(s) \Delta s \Delta u \\
& =\int_{t_{0}}^{t}\left\{\int_{\sigma(s)}^{t} r(t, u) a(u, s) \Delta u\right\} x(s) \Delta s \\
& =\int_{t_{0}}^{t}[r(t, s)+a(t, s)] x(s) \Delta s .
\end{aligned}
$$

Thus,

$$
-\int_{t_{0}}^{t} r(t, u) f(u) \Delta u=\int_{t_{0}}^{t} a(t, s) x(s) \Delta s,
$$

which implies (2.6). One may also show by using (2.7) that (2.6) implies (2.4).

Next, we consider the nonlinear system of integral equations of the form

$$
\tilde{x}(t)=f(t)+\int_{t_{0}}^{t} a(t, s)\{\tilde{x}(s)+G(s, \tilde{x}(s))\} \Delta s,
$$

where $G(t, \tilde{x})$ indicates the higher-order terms of $\tilde{x}$. If the solution $\tilde{x}$ of (2.8) is known, then Equation (2.8) can be rewritten as

$$
\tilde{x}(t)=F(t)+\int_{t_{0}}^{t} a(t, s) \tilde{x}(s) \Delta s
$$

where

$$
F(t)=f(t)+\int_{t_{0}}^{t} a(t, s) G(s, \tilde{x}(s)) \Delta s .
$$

If the existence of resolvent $r(t, s)$ is known, we get by (2.6) that

$$
\begin{aligned}
\tilde{x}(t)= & F(t)-\int_{t_{0}}^{t} r(t, s) F(s) \Delta s \\
= & f(t)+\int_{t_{0}}^{t} a(t, s) G(s, \tilde{x}(s)) \Delta s \\
& \quad-\int_{t_{0}}^{t} r(t, s)\left\{f(s)+\int_{t_{0}}^{s} a(s, u) G(u, \tilde{x}(u)) \Delta u\right\} \Delta s \\
= & f(t)-\int_{t_{0}}^{t} r(t, s) f(s) \Delta s+\int_{t_{0}}^{t} a(t, s) G(s, \tilde{x}(s)) \Delta s \\
& -\int_{t_{0}}^{t}\left\{\int_{\sigma(u)}^{t} r(t, s) a(s, u) \Delta s\right\} G(u, \tilde{x}(u)) \Delta u
\end{aligned}
$$




$$
\begin{aligned}
=f(t) & -\int_{t_{0}}^{t} r(t, s) f(s) \Delta s \\
& +\int_{t_{0}}^{t}\left\{a(t, u)-\int_{\sigma(u)}^{t} r(t, s) a(s, u) \Delta s\right\} G(u, \tilde{x}(u)) \Delta u \\
=f(t) & -\int_{t_{0}}^{t} r(t, s) f(s) \Delta s-\int_{t_{0}}^{t} r(t, u) G(u, \tilde{x}(u)) \Delta u .
\end{aligned}
$$

Thus, from (2.6) we obtain the variation of constants form of Equation (2.8) as follows:

$$
\tilde{x}(t)=x(t)-\int_{t_{0}}^{t} r(t, u) G(u, \tilde{x}(u)) \Delta u .
$$

By making use of (2.9), (2.4), and (2.6), one may easily verify that (2.10) implies (2.8).

In the next section, we study the existence of resolvent $r(t, s)$ corresponding to the linear system of integral equations (2.4). We also show by Theorems 3.6 and 3.8 that

$$
\int_{\sigma(s)}^{t} r(t, u) a(u, s) \Delta u=\int_{\sigma(s)}^{t} a(t, u) r(u, s) \Delta u .
$$

This will enable us to rewrite (2.5) as

$$
r(t, s)=-a(t, s)+\int_{\sigma(s)}^{t} a(t, u) r(u, s) \Delta u .
$$

\section{Existence of resolvents}

3.1. $L^{p}$ case. Let the set $\Omega$ be given by

$$
\Omega:=\left\{(t, s) \in I_{\mathbb{T}} \times I_{\mathbb{T}}: t_{0} \leq s \leq t \leq T\right\} .
$$

Henceforth, we let $1<p<\infty$ and assume that $1 / p+1 / q=1$. For any $n \times n$ matrix $A$ we denote by $|A|$ the matrix norm

$$
|A|=\sup _{|x| \leq 1}\|A x\|
$$

where $\|u\|$ indicates the vector norm of $u$. Let us define the functions

$$
A(t):=\int_{t_{0}}^{t}|a(t, s)|^{q} \Delta s, \quad B(t):=\int_{t}^{T}|a(s, t)|^{p} \Delta s, t \in I_{\mathbb{T}}
$$

and

$$
c(t, s):=\int_{s}^{t} A(u)^{p / q} \Delta u, \quad(t, s) \in \Omega .
$$

Next, we define a class of $n \times n$ matrix-valued functions $\alpha: \Omega \rightarrow \mathbb{R}^{n \times n}$ satisfying the following conditions.

(C.1) $\alpha(t, s)$ is measurable in $(t, s) \in \Omega$ with $\alpha(t, s)=0$ a.e. when $\sigma(s)>t$. 
(C.2) For almost all $t$ in $I_{\mathbb{T}}$, the integral $\int_{t_{0}}^{T}|\alpha(t, s)|^{q} \Delta s$ exists, and for almost all $s$ in $I_{\mathbb{T}}$, the integral $\int_{t_{0}}^{T}|\alpha(t, s)|^{p} \Delta t$ exists.

(C.3) The numbers $\int_{t_{0}}^{T}\left\{\int_{t_{0}}^{T}|\alpha(t, s)|^{q} \Delta s\right\}^{p / q} \Delta t$ and $\int_{t_{0}}^{T}\left\{\int_{t_{0}}^{T}|\alpha(t, s)|^{p} \Delta t\right\}^{q / p} \Delta s$ are both finite.

Definition 3.1. We say that an $n \times n$ matrix-valued function $\alpha(t, s)$ is of type $\left(L^{p}, T\right)$ if and only if the conditions (C.1)-(C.3) hold.

EXAmple 3.2. Any function $\alpha(t, s)$ which is continuous in $(t, s)$ for $(t, s) \in \Omega$ is of type $\left(L^{p}, T\right)$ for all $p>1$ and $T>t_{0}$.

Definition 3.3. An $n \times n$ matrix-valued function $\alpha(t, s)$ is said to be of type $\mathcal{L} \mathcal{L}_{p}$ if and only if it is of type $\left(L^{p}, T\right)$ for each $T>t_{0}$.

Let the kernel $a(t, s)$ be of type $\left(L^{p}, T\right)$. Define the sequence $\left\{r_{n}(t, s)\right\}_{n \in \mathbb{N}}$ by

$$
\begin{gathered}
r_{1}(t, s):=a(t, s), \\
r_{n+1}(t, s):=\int_{\sigma(s)}^{t} a(t, u) r_{n}(u, s) \Delta u
\end{gathered}
$$

for $(t, s) \in \Omega$ and $r_{n}(t, s)=0$ for $t_{0} \leq t<\sigma(s) \leq T$.

The following lemma plays a substantial role in obtaining inequality (3.7).

Lemma 3.4. Let $1<p<\infty$ and the kernel $a(t, s)$ be of type $\left(L^{p}, T\right)$. Then

$$
\frac{\left\{c(t, s)^{n}\right\}^{\Delta_{t}}}{n !} \geq A(t)^{p / q} \frac{c(t, s)^{n-1}}{(n-1) !}
$$

holds for all positive integers $n>1$ and $(t, s) \in \Omega$.

PROOF. We use the formula

$$
\left\{f^{n+1}(t)\right\}^{\Delta}=\left\{\sum_{k=0}^{n} f(t)^{k} f(\sigma(t))^{n-k}\right\} f^{\Delta}(t)
$$

(see [5, Exercise 1.23]). Since $a(t, s)$ is of type $\left(L^{p}, T\right)$, it is obvious that $c(t, s)$ is $\Delta$-differentiable in its both parameters and

$$
c^{\Delta_{t}}(t, s)=A(t)^{p / q}, \quad c^{\Delta_{s}}(t, s)=-A(s)^{p / q} .
$$

On the other hand, it follows from (3.2) that $c$ is increasing in $t$ and decreasing in $s$, that is,

$$
c(\sigma(t), s) \geq c(t, s) \quad \text { and } \quad c(t, \sigma(s)) \leq c(t, s)
$$


for all $(t, s) \in \Omega$. Thus, from (3.6) we obtain

$$
\begin{aligned}
\frac{\left\{c(t, s)^{n}\right\}^{\Delta_{t}}}{(n-1) !} & =\frac{1}{(n-1) !}\left\{\sum_{k=0}^{n-1} c(t, s)^{k} c(\sigma(t), s)^{n-k-1}\right\} c^{\Delta}(t, s) \\
& \geq \frac{1}{(n-1) !}\left\{\sum_{k=0}^{n-1} c(t, s)^{k} c(t, s)^{n-k-1}\right\} A(t)^{p / q} \\
& =n A(t)^{p / q} \frac{c(t, s)^{n-1}}{(n-1) !} .
\end{aligned}
$$

This completes the proof.

LEMMA 3.5. Let $1<p<\infty$ and the kernel $a(t, s)$ be of type $\left(L^{p}, T\right)$. Then for each positive integer $n \geq 1$, the function $r_{n}(t, s)$ is of type $\left(L^{p}, T\right)$. Moreover, for each nonnegative integer $n \geq 0$ and for $(t, s) \in \Omega$,

$$
\left|r_{n+2}(t, s)\right| \leq A(t)^{1 / q} B(s)^{1 / p}\left\{c(t, s)^{n} / n !\right\}^{1 / p}
$$

holds.

PROOF. If $t_{0} \leq t<\sigma(s) \leq T$ for some $(t, s) \in \Omega$, then $r_{n+2}(t, s)=0$ and (3.7) holds. Suppose that $\sigma(s) \leq t$ for all $(t, s) \in \Omega$. We proceed by induction. From Hölder's inequality we find that

$$
\begin{aligned}
\left|r_{2}(t, s)\right| & \leq \int_{\sigma(s)}^{t}|a(t, u)||a(u, s)| \Delta u \\
& \leq\left\{\int_{s}^{t}|a(t, u)|^{q} \Delta u\right\}^{1 / q}\left\{\int_{s}^{t}|a(u, s)|^{p} \Delta u\right\}^{1 / p} \\
& \leq A(t)^{1 / q} B(s)^{1 / p} .
\end{aligned}
$$

This shows that the kernel $r_{2}$ satisfies (3.7) and is of type $\left(L^{p}, T\right)$. Suppose that $r_{1}, r_{2}, \ldots, r_{n+1}$ are all kernels of type $\left(L^{p}, T\right)$ and that (3.7) holds for $n-1$. It follows from (3.4) and (3.5) that

$$
\begin{aligned}
\left|r_{n+2}(t, s)\right| & =\int_{\sigma(s)}^{t}|a(t, u)|\left|r_{n+1}(u, s)\right| \Delta u \\
& \leq\left\{\int_{s}^{t}|a(t, u)|^{q} \Delta u\right\}^{1 / q}\left\{\int_{s}^{t}\left|r_{n+1}(u, s)\right|^{p} \Delta u\right\}^{1 / p} \\
& \leq A(t)^{1 / q}\left\{B(s) \int_{s}^{t} A(u)^{p / q} \frac{c(u, s)^{n-1}}{(n-1) !} \Delta u\right\}^{1 / p} \\
& \leq A(t)^{1 / q} B(s)^{1 / p}\left\{\int_{s}^{t}\left[c(u, s)^{n} / n !\right]^{\Delta_{u}} \Delta u\right\}^{1 / p} \\
& =A(t)^{1 / q} B(s)^{1 / p}\left\{c(t, s)^{n} / n !\right\}^{1 / p}
\end{aligned}
$$


Thus (3.7) is satisfied. Using (3.1) and (3.7), we conclude that $r_{n+2}$ is of type $\left(L^{p}, T\right)$. The proof is complete.

THEOREM 3.6. If $1<p<\infty$ and the kernel $a(t, s)$ is of type $\left(L^{p}, T\right)$, then there exists a kernel $r(t, s)$ of type $\left(L^{p}, T\right)$ which solves the resolvent Equation (2.11) a.e. in $(t, s) \in \Omega$.

PROOF. Let $r_{n}$ be defined by (3.3) and (3.4). Let

$$
r(t, s):=-\sum_{n=1}^{\infty} r_{n}(t, s) \quad \text { for }(t, s) \in \Omega
$$

and $r(t, s)=0$ whenever $t_{0} \leq t<\sigma(s) \leq T$. From (3.7) we obtain

$$
|r(t, s)| \leq|a(t, s)|+A(t)^{1 / q} B(s)^{1 / p} \sum_{n=2}^{\infty}\left\{\alpha^{n} / n !\right\}^{1 / p},
$$

where

$$
\alpha=\int_{t_{0}}^{T} A(u)^{p / q} \Delta u
$$

For any $n>\alpha$,

$$
\left\{\frac{\alpha^{n+1} n !}{\alpha^{n}(n+1) !}\right\}^{1 / p}=\left\{\frac{\alpha}{n+1}\right\}^{1 / p}<1 .
$$

This implies that the series in (3.9) converges by the ratio test. Since $A(t), B(s)$, and $a(t, s)$ are finite, $r(t, s)$ is a.e. well defined and measurable in $(t, s)$ for $(t, s) \in \Omega$. By (3.9) we deduce that $r$ is of type $\left(L^{p}, T\right)$. Finally, we resort to the Lebesgue dominated convergence theorem to obtain

$$
\begin{aligned}
\int_{\sigma(s)}^{t} a(t, u) r(u, s) \Delta u & =\int_{\sigma(s)}^{t} a(t, u)\left\{-\sum_{n=1}^{\infty} r_{n}(u, s)\right\} \Delta u \\
& =-\sum_{n=1}^{\infty} \int_{\sigma(s)}^{t} a(t, u) r_{n}(u, s) \Delta u \\
& =-\sum_{n=1}^{\infty} r_{n+1}(t, s) \\
& =r(t, s)+a(t, s) .
\end{aligned}
$$

This shows that $r$ defined in (3.8) solves Equation (2.11).

LEMMA 3.7. If $a(t, s)$ is of type $\left(L^{p}, T\right)$, then for any positive integers $v$ and $w$ with $v+w=n+1$,

$$
r_{n+1}(t, s)=\int_{\sigma(s)}^{t} r_{w}(t, u) r_{v}(u, s) \Delta u
$$


PROOF. For $n=1$, the proof is trivial. Let (3.10) be true for $w_{0}+v_{0} \leq n, n \geq 1$. Given $v, w \geq 1$ with $v+w=n+1$, define

$$
I(w, v)=\int_{\sigma(s)}^{t} r_{w}(t, u) r_{v}(u, s) \Delta u .
$$

Then

$$
\begin{aligned}
I(w, v) & =\int_{\sigma(s)}^{t} r_{w}(t, u)\left\{\int_{\sigma(s)}^{u} a(u, z) r_{v-1}(z, s) \Delta z\right\} \Delta u \\
& =\int_{\sigma(s)}^{t} \int_{\sigma(z)}^{t} r_{w}(t, u) a(u, z) r_{v-1}(z, s) \Delta u \Delta z \\
& =\int_{\sigma(s)}^{t} r_{w+1}(t, z) r_{v-1}(z, s) \Delta z \\
& =I(w+1, v-1) .
\end{aligned}
$$

Hence, we arrive at

$$
I(1, n)=I(2, n-1)=I(3, n-3) \cdots=I(n, 1),
$$

which proves the result for $n+1$.

From the previous results in this section we proceed to the next result.

THEOREM 3.8. If a $(t, s)$ is a kernel of type $\mathcal{L} \mathcal{L}_{p}$, then there exists a kernel $r(t, s)$ of type $\mathcal{L L}_{p}$ such that $r$ satisfies both resolvent Equations (2.5) and (2.11) for almost all $(t, s)$ in the region $\Omega$.

PROOF. Let $r(t, s)$ be defined by (3.8). We know from Theorem 3.6 that $r(t, s)$ is of type $\left(L^{p}, T\right)$ for each $T>t_{0}$ and solves the resolvent Equation (2.11) a.e. in $(t, s) \in \Omega$. On the other hand, we get by (3.8) and (3.10) that

$$
\begin{aligned}
\int_{\sigma(s)}^{t} r(t, u) a(u, s) \Delta u & =\int_{\sigma(s)}^{t}\left\{-\sum_{n=1}^{\infty} r_{n}(t, u)\right\} a(u, s) \Delta u \\
& =-\sum_{n=1}^{\infty} \int_{\sigma(s)}^{t} r_{n}(t, u) a(u, s) \Delta u \\
& =-\sum_{n=1}^{\infty} r_{n+1}(t, s) \\
& =r(t, s)+a(t, s),
\end{aligned}
$$

where the Lebesgue dominated convergence theorem enables us to interchange summation and integration. Hence, $r(t, s)$ solves Equation (2.5). The proof is complete.

Open PRoblem. Show the existence of a resolvent $r(t, s)$ of type $\mathcal{L} \mathcal{L}_{1}$ solving the Equations (2.5) and (2.11). 


\subsection{Shift operators.}

Definition 3.9. Let $\mathbb{T}$ be a time scale and $t_{0} \in \mathbb{T}$ a fixed number such that there exist operators $\delta_{ \pm}:\left[t_{0}, \infty\right)_{\mathbb{T}} \times \mathbb{T} \rightarrow \mathbb{T}$ satisfying the following properties.

(P.1) The functions $\delta_{ \pm}$are strictly increasing with respect to their second arguments, that is, if $\left(T_{0}, t\right),\left(T_{0}, u\right) \in \mathcal{D}_{ \pm}:=\left\{(s, t) \in\left[t_{0}, \infty\right)_{\mathbb{T}} \times \mathbb{T}: \delta_{ \pm}(s, t) \in \mathbb{T}\right\}$, then

$$
T_{0} \leq t<u \text { implies } \delta_{ \pm}\left(T_{0}, t\right)<\delta_{ \pm}\left(T_{0}, u\right) .
$$

(P.2) If $\left(T_{1}, u\right),\left(T_{2}, u\right) \in \mathcal{D}_{-}$with $T_{1}<T_{2}$, then

$$
\delta_{-}\left(T_{1}, u\right)>\delta_{-}\left(T_{2}, u\right)
$$

and if $\left(T_{1}, u\right),\left(T_{2}, u\right) \in \mathcal{D}_{+}$with $T_{1}<T_{2}$, then

$$
\delta_{+}\left(T_{1}, u\right)<\delta_{+}\left(T_{2}, u\right) .
$$

(P.3) If $t \in \mathbb{T}$, then $\left(t_{0}, t\right) \in \mathcal{D}_{ \pm}$and $\delta_{ \pm}\left(t_{0}, t\right)=t$ and if $t \in\left[t_{0}, \infty\right) \mathbb{T}$, then $\left(t, t_{0}\right) \in \mathcal{D}_{+}$ and $\delta_{+}\left(t, t_{0}\right)=\delta_{+}\left(t_{0}, t\right)=t$.

(P.4) If $(s, t) \in \mathcal{D}_{+}$, then $\left(s, \delta_{+}(s, t)\right) \in \mathcal{D}_{-}$and $\delta_{-}\left(s, \delta_{+}(s, t)\right)=t$.

(P.5) If $(s, t) \in \mathcal{D}_{+}$and $\left(u, \delta_{+}(s, t)\right) \in \mathcal{D}_{-}$, then $\left(s, \delta_{-}(u, t)\right) \in \mathcal{D}_{+}$and

$$
\delta_{-}\left(u, \delta_{+}(s, t)\right)=\delta_{+}\left(s, \delta_{-}(u, t)\right) .
$$

The operators $\delta_{-}$and $\delta_{+}$associated with the $t_{0} \in \mathbb{T}$ (called an initial point) are said to be backward and forward shift operators on $\mathbb{T}$, respectively. The variable $s \in\left[t_{0}, \infty\right)_{\mathbb{T}}$ in $\delta_{ \pm}(s, t)$ is called the shift size. The values $\delta_{+}(s, t)$ and $\delta_{-}(s, t)$ in $\mathbb{T}$ denote translation of $t \in \mathbb{T}$ by $s$ units to the right and left, respectively. The sets $\mathcal{D}_{ \pm}$ are the domains of the shift operators $\delta_{ \pm}$, respectively.

Notice that (P.3)-(P.5) imply $\delta_{-}(u, u)=\delta_{-}\left(u, \delta_{+}\left(u, t_{0}\right)\right)=t_{0}$, and hence, $(u, u) \in$ $\mathcal{D}_{\text {_ for all }} u \in\left[t_{0}, \infty\right)_{\mathbb{T}}$.

Definition 3.10. A point $t^{*} \in \mathbb{T}$ is said to be a sticky point if

$$
\delta_{ \pm}\left(s, t^{*}\right)=t^{*} \quad \text { for all } s \in\left[t_{0}, \infty\right) \mathbb{T} \text { with }\left(s, t^{*}\right) \in \mathcal{D}_{ \pm} .
$$

Henecforth, we will denote by $\mathbb{T}^{*}$ the largest subset of $\mathbb{T}$ without a sticky point.

Corollary 3.11. If $t_{0} \in \mathbb{T}^{*}$, then $t^{*} \notin\left[t_{0}, \infty\right) \mathbb{T}$.

Proof. If $t_{0} \in \mathbb{T}^{*}$ and $t^{*} \in\left[t_{0}, \infty\right)_{\mathbb{T}}$ is a sticky point, then

$$
t^{*}=\delta_{-}\left(t^{*}, t^{*}\right)=t_{0} \in \mathbb{T}^{*}=\mathbb{T}-\left\{t^{*}\right\}
$$

This leads to a contradiction. 
EXAMPLE 3.12. Let $\mathbb{T}=\mathbb{R}$ and $t_{0}=1$. The operators

$$
\delta_{-}(s, t)=\left\{\begin{array}{ll}
t / s & \text { if } t \geq 0 \\
s t & \text { if } t<0,
\end{array} \quad \text { for } s \in[1, \infty)\right.
$$

and

$$
\delta_{+}(s, t)=\left\{\begin{array}{ll}
s t & \text { if } t \geq 0 \\
t / s & \text { if } t<0,
\end{array} \quad \text { for } s \in[1, \infty)\right.
$$

are left and right shift operators associated with the initial point $t_{0}=1$. Also, $t^{*}=0$ is a sticky point (that is, $\mathbb{T}^{*}=\mathbb{R}-\{0\}$ ) since

$$
\delta_{ \pm}(s, 0)=0 \quad \forall s \in[1, \infty) .
$$

In the following table we give some time scales with their corresponding shift operators (note that $\mathbb{N}^{1 / 2}=\{\sqrt{n}: n \in \mathbb{N}\}$ and $q^{\mathbb{Z}}=\left\{q^{n}: n \in \mathbb{Z}\right.$ and $\left.q>1\right\}$ ).

\begin{tabular}{cccccc}
\hline $\mathbb{T}$ & $t_{0}$ & $t^{*}$ & $\mathbb{T}^{*}$ & $\delta_{-}(s, t)$ & $\delta_{+}(s, t)$ \\
\hline $\mathbb{R}$ & 0 & N/A & $\mathbb{R}$ & $t-s$ & $t+s$ \\
$\mathbb{Z}$ & 0 & N/A & $\mathbb{Z}$ & $t-s$ & $t+s$ \\
$q^{\mathbb{Z}} \cup\{0\}$ & 1 & 0 & $q^{\mathbb{Z}}$ & $t / s$ & $s t$ \\
$\mathbb{N}^{1 / 2}$ & 0 & N/A & $\mathbb{N}^{1 / 2}$ & $\sqrt{t^{2}-s^{2}}$ & $\sqrt{t^{2}+s^{2}}$ \\
\hline
\end{tabular}

Definition 3.9 yields the following result.

LEMMA 3.13. Let $\delta_{-}$and $\delta_{+}$be the shift operators associated with the initial point $t_{0}$. Then:

(i) $\delta_{+}(s, t)=u$ if and only if $\delta_{-}(s, u)=t$;

(ii) $\delta_{+}(s, t) \in\left[t_{0}, \infty\right)_{\mathbb{T}}$ for all $(s, t) \in \mathcal{D}_{+}$with $t \geq t_{0}$;

(iii) $\delta_{-}(s, t) \in\left[t_{0}, \infty\right)_{\mathbb{T}}$ for all $(s, t) \in \mathcal{D}_{-}$with $t \geq s$;

(iv) if $\delta_{+}(s, t)$ is $\Delta$-differentiable in its second variable $t \in \mathbb{T}_{\kappa}^{*}$, then $\delta_{+}^{\Delta_{t}}(s, t)>0$ for all $(s, t) \in \mathcal{D}_{+}$with $t \in \mathbb{T}_{\kappa}^{*}$.

PROOF. (i) is obtained from (P.4). (ii) and (iii) follow from (P.1)-(P.2). Since $\delta_{+}(s, t)$ is strictly increasing in its second variable, we have (iv) by [6, Corollary 1.16].

Notice that the shift operators $\delta_{ \pm}$are defined once the initial point $t_{0} \in \mathbb{T}$ is known. For instance, we choose the initial point $t_{0}=0$ to define shift operators $\delta_{ \pm}(s, t)=$ $t \pm s$ on $\mathbb{T}=\mathbb{R}$. However, if we take the initial point $\lambda \in(0, \infty)$ then we can define new shift operators by $\widetilde{\delta}_{ \pm}(s, t)=t \mp \lambda \pm s$ and in terms of $\delta_{ \pm}$as

$$
\widetilde{\delta}_{ \pm}(s, t)=\delta_{\mp}\left(\lambda, \delta_{ \pm}(s, t)\right) .
$$

EXAMPLE 3.14. In the following table, we give several time scales to show the change in the formula of shift operators as the initial point changes. 


\begin{tabular}{cccccccc}
\hline & \multicolumn{2}{c}{$\mathbb{T}=\mathbb{N}^{1 / 2}$} & \multicolumn{2}{c}{$\mathbb{T}=h \mathbb{Z}$} & \multicolumn{2}{c}{$\mathbb{T}=2^{\mathbb{N}}$} \\
\hline$t_{0}$ & 0 & $\lambda$ & 0 & $h \lambda$ & 1 & $2^{\lambda}$ \\
$\delta_{-}(s, t)$ & $\sqrt{t^{2}-s^{2}}$ & $\sqrt{t^{2}+\lambda^{2}-s^{2}}$ & $t-s$ & $t+h \lambda-s$ & $t / s$ & $2^{\lambda} t s^{-1}$ \\
$\delta_{+}(s, t)$ & $\sqrt{t^{2}+s^{2}}$ & $\sqrt{t^{2}-\lambda^{2}+s^{2}}$ & $t+s$ & $t-h \lambda+s$ & $t s$ & $2^{-\lambda} t s$ \\
\hline
\end{tabular}

Here $\lambda \in \mathbb{Z}_{+}, 2^{\mathbb{N}}=\left\{2^{n}: n \in \mathbb{N}\right\}$, and $h \mathbb{Z}=\{h n: n \in \mathbb{Z}\}$.

3.3. The continuous case. Without the notion of a shift operator, one would have to require that $t+s$ and $t-s$ be in the time scale. We decided this was an unnecessary requirement that we overcame with the creation of the shift operator. Also, we expect researchers to make use of this new notion to overcome such difficulties.

In this section, we are concerned with the existence of the continuous function $r(t, s)$ on $\Omega$ which solves the resolvent Equations (2.5) and (2.11).

Let $\mathbb{T}$ be a time scale containing an initial point $t_{0} \in \mathbb{T}^{*}$ so that there exist the shift operators $\delta_{ \pm}$associated with $t_{0}$. Hereafter, we shall suppose that the right shift operator $\delta_{+}(s, t)$ on $\mathbb{T}$ is $\Delta$-differentiable in its second variable with $r d$-continuous derivative. Substituting $\delta_{+}(\sigma(s), t)$ for $t$ in Equation (2.11), we get

$$
r\left(\delta_{+}(\sigma(s), t), s\right)=-a\left(\delta_{+}(\sigma(s), t), s\right)+\int_{\sigma(s)}^{\delta_{+}(\sigma(s), t)} a\left(\delta_{+}(\sigma(s), t), v\right) r(v, s) \Delta v .
$$

Using (3.11) and [5, Theorem 1.98], we obtain

$$
\begin{aligned}
r\left(\delta_{+}(\sigma(s), t), s\right) & \\
= & -a\left(\delta_{+}(\sigma(s), t), s\right) \\
& +\int_{t_{0}}^{t} a\left(\delta_{+}(\sigma(s), t), \delta_{+}(\sigma(s), u)\right) \delta_{+}^{\Delta_{u}}(\sigma(s), u) r\left(\delta_{+}(\sigma(s), u), s\right) \Delta u .
\end{aligned}
$$

The advantage of considering a resolvent equation of the form (3.12) is that the variable $s$ appears in the equation only as a parameter. If the role of $s$ is suppressed then (3.12) turns into

$$
R(t)=-A(t)+\int_{t_{0}}^{t} a_{s}(t, u) R(u) \Delta u,
$$

where $R(t)=r\left(\delta_{+}(\sigma(s), t), s\right), A(t)=a\left(\delta_{+}(\sigma(s), t), s\right)$ and

$$
a_{s}(t, u)=a\left(\delta_{+}(\sigma(s), t), \delta_{+}(\sigma(s), u)\right) \delta_{+}^{\Delta_{u}}(\sigma(s), u) \quad \text { for }(t, s) \in \Omega .
$$

Let $\beta>0$ be a constant and let $\|$.$\| denote the Euclidean norm on \mathbb{R}^{n}$. Define $J_{\mathbb{T}}:=[a, b]_{\mathbb{T}}$. We will consider the space $C\left(J_{\mathbb{T}}, \mathbb{R}^{n}\right)$ of continuous functions endowed with a suitable norm

$$
\|x\|_{\beta}:=\sup _{t \in J_{\mathbb{T}}} \frac{\|x(t)\|}{e_{\beta}(t, a)} \quad \text { or } \quad\|x\|_{0}:=\sup _{t \in J_{\mathbb{T}}}\|x(t)\| .
$$


It was proven in [11, Lemma 3.3] that $\|x\|_{\beta}$ is a norm and is equivalent to the supremum norm $\|x\|_{0}$. Also, it was concluded that $\left(C\left(J_{\mathbb{T}}, \mathbb{R}^{n}\right),\|x\|_{\beta}\right)$ is a Banach space. We will denote this Banach space by $\mathcal{P}$.

To prove the next result we will employ the following theorem.

THEOREM 3.15 [9, Theorem 3.4]. Consider the linear integral equation

$$
x(t)=f(t)+\int_{\alpha}^{t} B(t, s) x(s) \Delta s, \quad t \in J_{\mathbb{T}} .
$$

Let $B: J_{\mathbb{T}} \times J_{\mathbb{T}} \rightarrow \mathbb{R}^{n \times n}$ be a matrix-valued function which is continuous in its first variable and rd-continuous in its second variable and let $f: J_{\mathbb{T}} \rightarrow \mathbb{R}^{n}$ be continuous. Then (3.14) has a unique solution in $\mathcal{P}$. In addition, if a sequence of functions $\left\{x_{i}\right\}$ is defined inductively by choosing any $x_{0} \in C\left(I_{\mathbb{T}}, \mathbb{R}^{n}\right)$ and setting

$$
x_{i+1}(t)=f(t)+\int_{\alpha}^{t} B(t, s) x_{i}(s) \Delta s, \quad t \in J_{\mathbb{T}},
$$

then the sequence $\left\{x_{i}\right\}$ converges uniformly on $I_{\mathbb{T}}$ to the unique solution $x$ of (3.14).

THEOREM 3.16. If $a(t, s)$ is continuous in $(t, s)$ for $(t, s) \in \Omega$, then there exists a continuous function $r(t, s)$ on $\Omega$ which solves the resolvent Equations (2.5) and (2.11).

PROOF. We can conclude from Theorem 3.15 that for each fixed $s \geq 0$ there exists a unique solution $R(t ; s)$ of (3.13) on $t_{0} \leq t \leq \delta_{-}(\sigma(s), T)$. Moreover, $R(t ; s)$ is continuous in the pair $(t, s)$. Let us define

$$
r(t, s)=R\left(\delta_{-}(\sigma(s), t) ; s\right) \quad \text { for }(t, s) \in \Omega .
$$

Evidently, $r$ solves (2.11). Since $a$ is continuous in $(t, s)$ for $(t, s) \in \Omega$, from Theorem 3.8 there is an $L^{2}$ function $k(t, s)$ which solves (2.5) and (2.11). By the uniqueness of the resolvent $k(t, s)$ among $L^{2}$ functions, $k=r$. Thus, $r$ also solves Equation (2.5).

\section{References}

[1] M. Bohner and O. Doslý, 'Oscillation of symplectic dynamic systems', ANZIAM J. 46 (2004), $17-32$.

[2] M. Bohner and G. Guseinov, 'Riemann and Lebesgue integration', Advances in Dynamic Equations on Time Scales (Birkhäuser, Boston, MA, 2003).

[3] M. Bohner and G. Guseinov, 'Multiple integration on time scales', Dynam. Systems Appl. 14(3-4) (2005), 579-606.

[4] M. Bohner and G. Guseinov, 'Double integral calculus of variations on time scales', Comput. Math. Appl. 54(1) (2007), 45-57.

[5] M. Bohner and A. Peterson, 'Dynamic equations on time scales', An Introduction with Applications (Birkhäuser, Boston, MA, 2001).

[6] M. Bohner and A. Peterson, Advances in Dynamic Equations on Time Scales (Birkhäuser, Boston, MA, 2003). 
[7] S. Hilger, 'Analysis on measure chains: a unified approach to continuous and discrete calculus', Results Math. 18 (1990), 18-56.

[8] B. Karpuz, 'Unbounded oscillation of higher-order nonlinear delay dynamic equations of neutral type with oscillating coefficients', Electron J. Qual. Theory Differ. Equ. 34(1-14) (2009).

[9] T. Kulik and C. C. Tisdell, 'Volterra integral equations on time scales: basic qualitative and quantitative results with applications to initial value problems on unbounded domains', Int. J. Difference Equ. 3(1) (2008), 103-133.

[10] R. K. Miller, Nonlinear Volterra Integral Equations (W. A. Benjamin, Menlo Park, CA, 1971).

[11] C. C. Tisdell and A. Zaidi, 'Basic qualitative and quantitative results for solutions to nonlinear, dynamic equations on time scales with an application to economic modelling', Nonlinear Anal. 68(11) (2008), 3504-3524.

[12] A. Zafer, 'The exponential of a constant matrix on time scales', ANZIAM J. 48 (2006), 99-106.

MURAT ADIVAR, Izmir University of Economics, Department of Mathematics, 35330 Izmir, Turkey

e-mail: murat.adivar@ieu.edu.tr

YOUSSEF N. RAFFOUL, University of Dayton, Department of Mathematics, Dayton, OH 45469-2316, USA

e-mail: youssef.raffoul@notes.udayton.edu 